\title{
A CASE OF KARTAGENER SYNDROME
}

Pagadpally Srinivas

1. Assistant Professor. Department of Paediatrics, Vinayaka Missions Medical College, Karaikal

\section{CORRESPONDING AUTHOR:}

Pagadpally Srinivas, 72, Vellai Pillaiyar Koil Street, Opposite government girls high school, Kottucherry, Karaikal- 609609.

E-mail: sreenu77@gmail.com

Ph: 00919994854115.

INTRODUCTION: Kartagener syndrome is a inherited disorder characterised by impaired ciliary dysfunction due to primary ciliary dyskinesia(PCD) along with situs inversus, leading to diverse clinical manifestations like chronic sinopulmonary infection, persistent middle ear infection and infertility. We report a young male child with chronic sinopulmonary infection and situs inversus.

KEY WORDS: Kartagener, primary ciliary dyskinesia, Situs inversus.

CASE REPORT: A 10 year old boy presented to our outpatient department with complaints of productive cough, repeated sinus infection, ear discharge and hard of hearing since the past few months. He was having similar complaints since the past few years. He was taking treatment at various hospitals for the same. He was born to consanguineous parents and there were no similar complaints in any other family members. On examination, oral cavity was congested and ear showed bilaterally retracted tympanic membrane. Height and weight were normal. During auscultation of the chest, the heart sounds were noted to be more prominent on the right side. On palpation of the chest, cardiac impulse was noted to be on the right side of the chest in the $4^{\text {th }}$ Intercostal space in the midclavicular line. On palpation of the abdomen, liver border could not be noted on the right hypochondria. $\mathrm{X}$ ray chest with abdomen was taken which confirmed dextrocardia and situs inversus. There was no clinical or radiological evidence of bronchiectasis. ECG revealed dextrocardia. USG abdomen revealed situs inversus totalis. Echocardiography confirmed situs inversus.

Otorhinology consultation was taken. X ray PNS showed pansinusitis and a diagnosis of chronic sinusitis was made after examination. Saccharine test was done, which revealed borderline delayed mucociliary clearance time. Considering the clinical picture of the chronic bronchitis, sinusitis, and situs inversus, the clinical diagnosis of Kartagener's syndrome was made. Child was started on oral antibiotics for sinusitis and advised to get pneumococcal vaccine, H.influenza vaccine. HRCT was planned at a later date as there was no clinical evidence of bronchiectasis.

DISCUSSION: First described by Siewert and later by Swiss paediatrician Manes (1897-1975), Kartagener published his description of Kartagener's syndrome in 1933.

Primary Ciliary Dyskinesia (PCD), also known as Immotile Cilia Syndrome, is a rare disease characterized by alteration of structure and function of cilia at the surface of respiratory epithelial cells and of the sperm tail. The defect mainly results in impaired mucociliary transport 
in the upper airways and infertility. Kartagener syndrome is a triad of Primary ciliary dyskinesia, chronic bronchitis/bronchiectasis and situs inversus.

Primary ciliary dyskinesia is an autosomal recessive disorder with incomplete penetrance [1]. The prevalence of PCD in children with respiratory disease is as high as $5 \%$ affecting 1 in 12,000to 1 in 20,000 live births [2].PCD also called immotile cilia syndrome is characterised by congenital abnormalities of ciliary function and structure [3, 4]. Cilia line the nose, paranasal sinuses, middle ear, Eustachian tube, bronchi as far as respiratory bronchioles and tail of spermatozoa. Normal ciliary function is critical in respiratory host defence and motility of sperms. Impairment of ciliary clearance at all the sites leads to the characteristic signs and symptoms of chronic sinusitis, chronic bronchitis eventually leading to bronchiectasis, often serous otitis media. Normal cilia are $6 \mu \mathrm{m}$ in length and beat 10 to 15 times per second with a fast forward and slower recovery stroke. They are composed of central axonemes containing 9 pairs of micro tubules that form a circle around 2 central micro tubules. Each of the 9 doublets has an outer and inner dynein arms. Dynein proteins are the force producing elements in cilia and acquire ATP as energy source. Most common abnormalities are absence of dynein arms, radial spokes or micro tubules. These patients are dependent on cough for clearance. $50 \%$ of these patients have dextrocardia (Kartagener syndrome) which is thought to be due to abnormal cellular micro tubules which are involved in rotation of organs in embryo.PCD is a genetically heterogenous disorder involving multiple genes most of which occur in genes coding for dynein proteins. The candidate genes involved include DNAI1 and DNAH5 [2, 5]. DNAI1 is a large gene located on chromosome 9 (9p 13-21) is highly expressed in trachea and testis. DNAH5 gene is localised to chromosome 5p (5p 14-15) expressed in lung, kidneys, brain and testis. Additional genes DNAH11, DNAI2 are also implicated.

Most patients with PCD present in the newborn period shortly after birth with respiratory distress, which manifests as tachypnea, hypoxemia, or even respiratory failure requiring mechanical ventilation. Clinical features may begin early in the new born period or become apparent during $2^{\text {nd }}$ to $3^{\text {rd }}$ decade of life. Infants and children present with history of recurrent otitis media, chronic bronchitis, and chronic sinusitis. Bacterial cultures of sputum or bronchial aspirates commonly yield non typable Haemophilus influenza, Staphylococcus aureus, Streptococcus pneumoniae, and Pseudomonas aeruginosa. Persistent airway infection and inflammation lead to bronchiectasis, even in preschool children. Clubbing is a sign of longstanding pulmonary involvement. Recurrent fever, haemoptysis and digital clubbing may also be present. Recurrent otitis media may lead to conductive hearing loss. Left-right laterality defects are found in PCD; 50\% of patients have situs inversus totalis with transposition of the thoracic and abdominal organs. Although sperm counts may be normal, most males are infertile and $50 \%$ of females are infertile because of reduced motility of ova in the fallopian tubes ${ }^{[4]}$. A few cases may also have hydrocephalus [6] and retinitis pigmentosa. Siblings of Kartagener's have pan sinusitis and bronchitis without situs inversus.

Currently diagnosis requires presence of characteristic phenotype and ultra structural defects of cilia. It should be suspected in any child with chronic or recurrent URI or LRI, Average age of diagnosis is 4 years. High index of suspicion is needed. Situs inversus totalis in a child who has chronic respiratory symptoms is virtually diagnostic of Kartagener's syndrome. Imaging studies show extensive involvement of PNS. Chest radiograph may show dextrocardia, bilateral lung poor infiltration and peribronchial infiltrates. USG abdomen may reveal right left disorientation. CT scan may reveal bronchiectasis. Spirometry reveals obstructive airway disease. Simple screening tests are performed by microscopic examination of nasal epithelium 
or by the saccharine test. Microscopic examination of scrapings of nasal epithelium (middle to posterior $3^{\text {rd }}$ of nose and placed into tissue culture medium). Absence of ciliary beat is presumptive of diagnosis of PCD. Impaired ciliary clearance may be detected by presence of abnormal saccharine test, in which time is recorded for saccharine to be tasted in the mouth after a $0.5 \mathrm{~mm}$ particle is kept in the inferior turbinate of nose. Usually it takes about 30 minutes but times up to $1 \mathrm{hr}$ may be normal. Diagnosis in adults is done by sperm examination and checking the motility of sperms.

Transmission electron microscopy is the current gold standard to assess ultrastructural defects, shortening or absence of dynein arms is the most common finding. Genetic testing for PCD is available for specific DNAI1 and DNAH5 mutations.

Treatment: No specific treatment will alter the ciliary function in PCD.

Bronchopulmonary disease is treated by chest physiotherapy, antibiotics and bronchodilators. Bronchiectasis may involve excision of involved areas. Gene therapy may be anticipated in the foreseeable future. Prognosis: Majority of the children develop chronic bronchiectasis in childhood. Bronchiectasis may present during $3^{\text {rd }}$ to $4^{\text {th }}$ decade of life.

\section{REFERENCES:}

1. Bush A, Cole PT, Hariri M et al, Primary ciliary dyskinesia - diagnosis and standards of care. Eurrespi J 1998;12:982-988

2. Robert Kliegman, Bonita F Stanton, Joseph W St.Geme, Nina F Schor, Richard E Behrman In: Nelson Textbook of Pediatrics (19 ${ }^{\text {th }}$ edition). Elsevier Saunders, Philadelphia. Web edition. www.expertconsult.com

3. Afzeius BA. A human syndrome caused by immotile cilia. Science 1976; 193: 317-319

4. Afzeius BA. Immotile cilia syndrome: Past, present and future prospects. Thorax 1998; 53: $143-144$

5. Olbrich $\mathrm{H}$, Haffner $\mathrm{K}$, Kispert A, et al: Mutations in DNAH5 cause primary ciliary dyskinesia and randomization of left-right asymmetry. Nat Genet 2002;30: 143-44.

6. Al-Shroof M, Karnik AM, Karnik AA, et al: Ciliary dyskinesia associated with hydrocephalus and mental retardation in a Jordanian family. Mayo ClinProc 2001;76:1219-24.

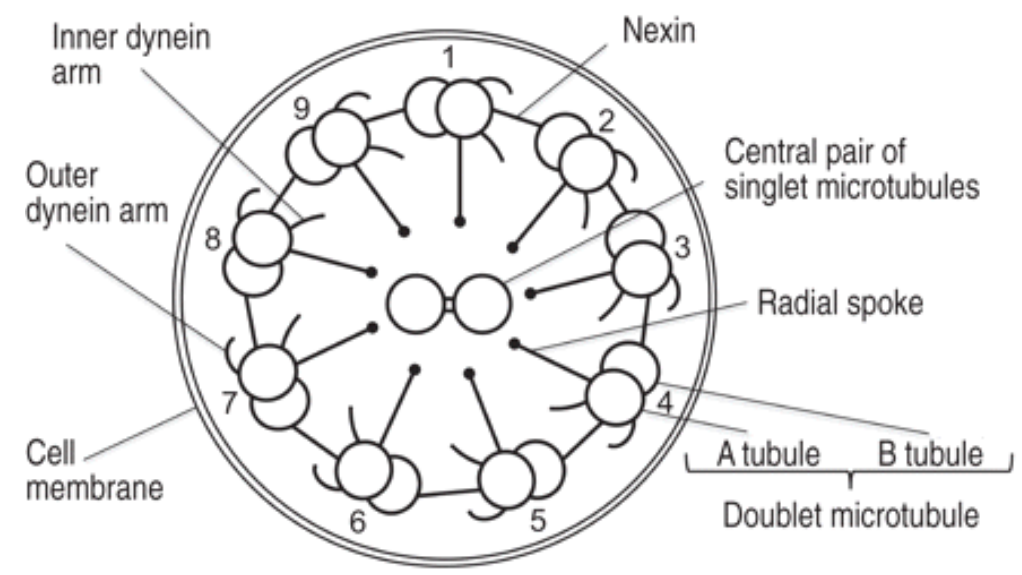

Figure 1: Normal respiratory cilia. 


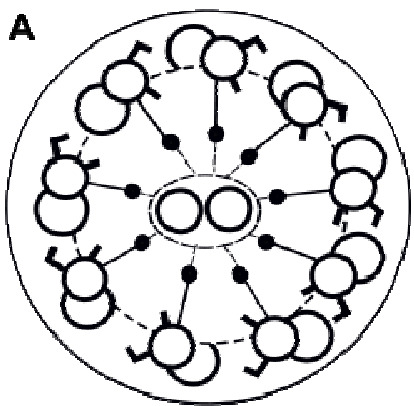

normal cilia

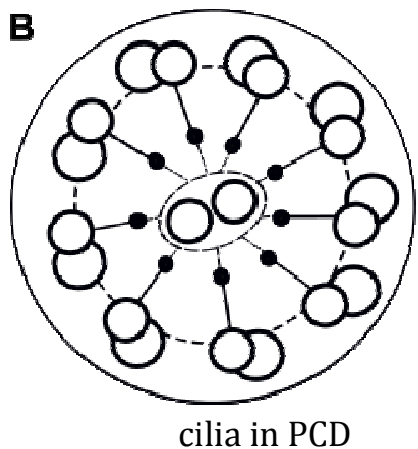

cilia in PCD

Figure 2: Comparison of a normal cilia and in PCD.

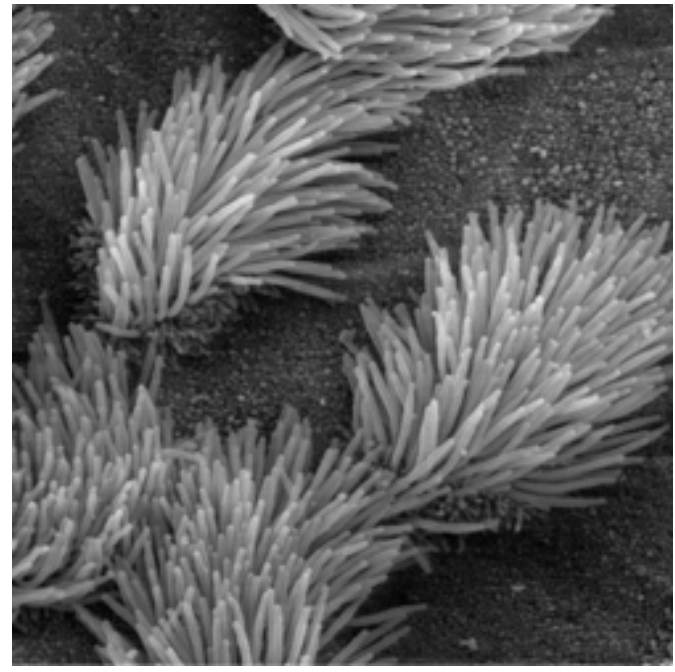

Figure 3: Electron microscope view of respiratory cilia.

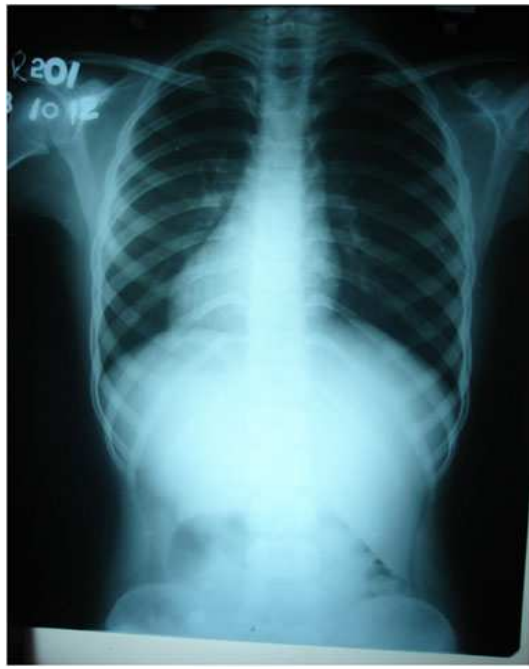

Figure $4 \mathrm{X}$ Ray chest with abdomen shows dextrocardia and liver on the left side

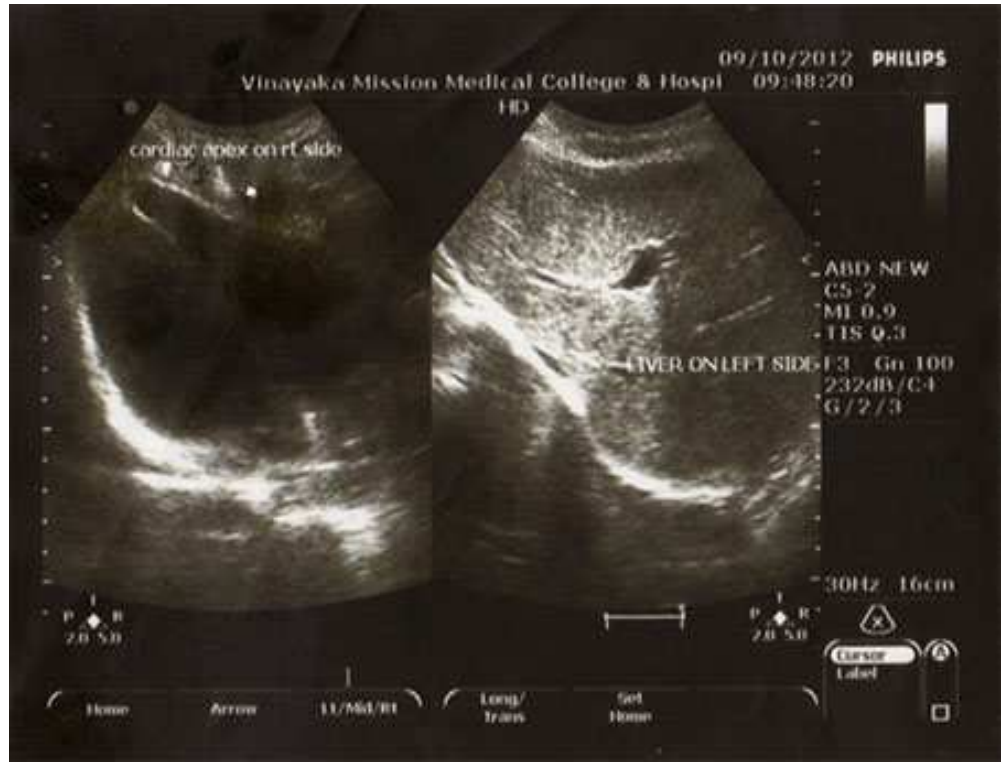

Figure 5 USG Abdomen showing situs inversus 


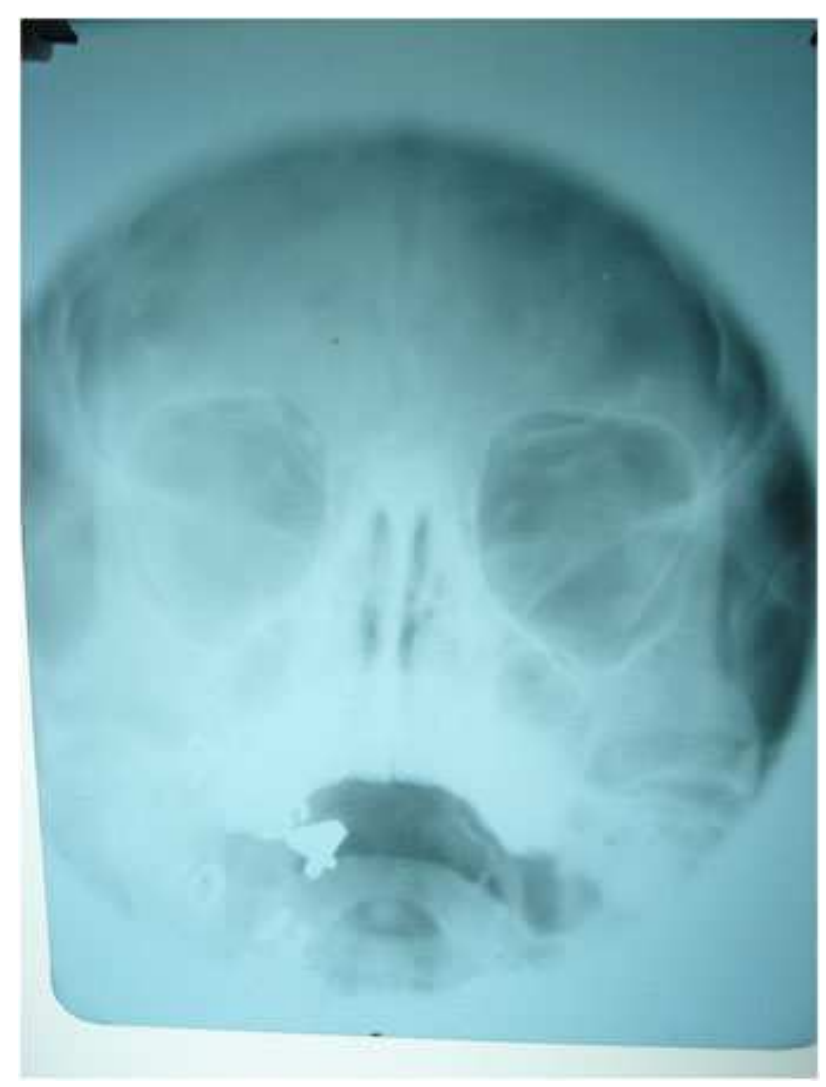

Figure 6 X Ray PNS showing pansinusitis

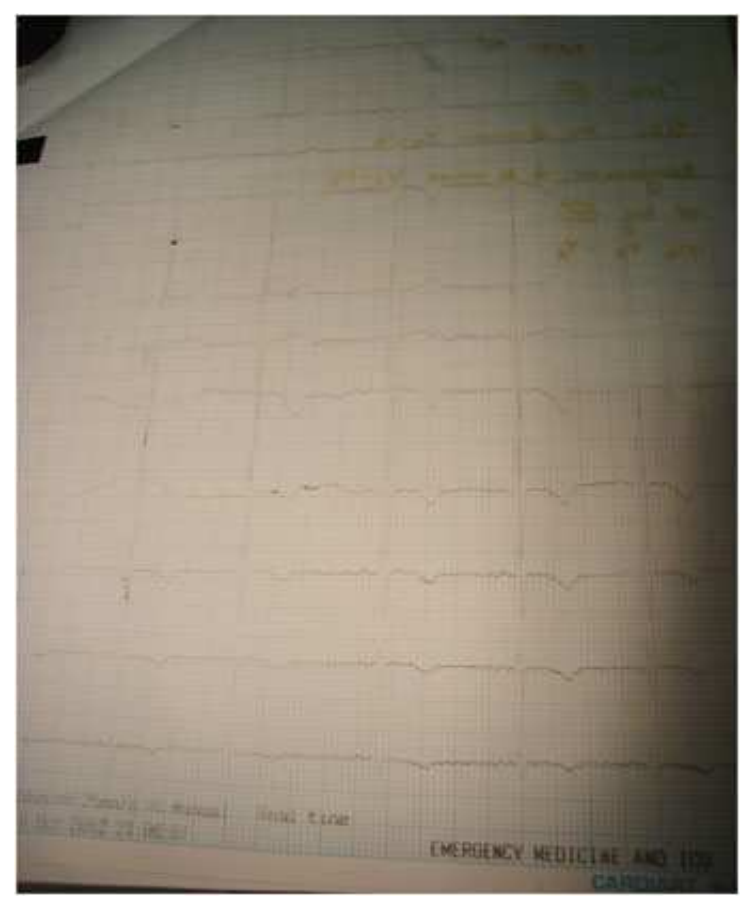

Figure 7 ECG showing typical changes of dextrocardia. 